\title{
An Euler-Lagrange Equation Only Depending on Derivatives of Caputo for Fractional Variational Problems with Classical Derivatives
}

\author{
Melani Barrios ${ }^{1,2, *}$, Gabriela Reyero ${ }^{1, *}$ \\ ${ }^{1}$ Departamento de Matemática, Facultad de Ciencias Exactas, Ingeniería y Agrimensura, Universidad Nacional de Rosario, Argentina \\ ${ }^{2}$ CONICET, Departamento de Matemática, Facultad de Ciencias Exactas, Ingeniería y Agrimensura, Universidad Nacional de Rosario, \\ Argentina
}

\begin{abstract}
In this paper we present advances in fractional variational problems with a Lagrangian depending on Caputo fractional and classical derivatives. New formulations of the fractional Euler-Lagrange equation are shown for the basic and isoperimetric problems, one in an integral form, and the other that depends only on the Caputo derivatives. The advantage is that Caputo derivatives are more appropriate for modeling problems than the Riemann-Liouville derivatives and makes the calculations easier to solve because, in some cases, its behavior is similar to the behavior of classical derivatives. Finally, a new exact solution for a particular variational problem is obtained.
\end{abstract}

Keywords Fractional Derivatives and Integrals, Fractional Variational Problems, Euler-Lagrange Fractional Equations, Fractional Isoperimetric Problems

AMS 2010 subject classifications 26A33, 49J99, 49K99

DOI: $10.19139 /$ soic-2310-5070-865

\section{Introduction}

The fractional variational calculus is a recent field, started in 1997, where classical variational problems are considered, but in the presence of fractional derivatives or integrals. In the last years numerous works have been developed tending to extend the theory of the variational calculus [35] in order to be able to be applied to problems of fractional variational calculus $[1,8,10,11,18,23]$. This is fundamentally due, on the one hand, to an important development of the fractional calculus both from the mathematical point of view and its applications in other areas (electricity, magnetism, mechanics, dynamics of fluids, medicine, etc) [7, 17, 19, 20, 22]; which has led to an overwhelming growth of its study in recent decades. On the other hand, the fractional differential equations establish models far superior to those that use differential equations with integer derivatives because they incorporate into the model issues of memory or later effects that are neglected in the models with classical derivative $[9,15,24,33]$.

Following [31], we consider here that the highest derivative in the Lagrangian is of integer order. The main advantage of our formulation, with respect to the pure fractional approach adopted in the literature, is that the classical results of variational calculus can now be obtained as particular cases. We recall that the only possibility to obtain the classical derivative $y^{\prime}$ from a fractional derivative $D^{\alpha} y, \alpha \in(0,1)$, is to take the limit when $\alpha$ tends to one. However, in general such a limit does not exist [34]. Different from [31], where the fractional derivative are considered in the sense of Riemann-Liouville, here we investigate problems of the calculus of variations with

\footnotetext{
${ }^{*}$ Correspondence to: Melani Barrios (Email: melani@ fceia.unr.edu.ar), Gabriela Reyero (Email: greyero@ @ceia.unr.edu.ar). Departamento de Matemática, Facultad de Ciencias Exactas, Ingeniería y Agrimensura, Universidad Nacional de Rosario, Avda. Pellegrini 250, S2000BTP Rosario, Argentina.
}

ISSN 2310-5070 (online) ISSN 2311-004X (print)

Copyright (c) 2020 International Academic Press 
integrands depending on the independent variable $x$, an unknown function $y$, its integer order derivative $y^{\prime}$, and a fractional derivative ${ }^{C} D^{\alpha} y$ in the sense of Caputo.

Recently, several approaches have been developed in order to generalize the Euler-Lagrange equations to include fractional derivatives $[2,3,4,5,6,12,13,16,26,27,28,31]$. In all the above mentioned references, the EulerLagrange equations always depend on the Riemann-Liouville or mixed Caputo and Riemann-Liouville derivatives. For the state of the art of the fractional calculus of variations and respective fractional Euler-Lagrange equations, see [29].

It is important to remark that while the Riemann-Liouville fractional derivatives [32] are historically the most studied approach to fractional calculus, the Caputo [14] approach to fractional derivatives is the most popular among physicists and scientists, because the differential equations defined in terms of Caputo derivatives require regular initial and boundary conditions. Furthermore, differential equations with Riemann-Liouville derivatives require nonstandard fractional initial and boundary conditions that lead, in general, to singular solutions, thus limiting their application in physics and science [21,22]. On the other hand, within current formulations of the fractional calculus of variations, even Lagrangians depending only on Caputo derivatives lead to Euler-Lagrange equations with Riemann-Liouville derivatives [6]. This is a consequence of the Lagrange method to optimize functionals: application of integration by parts for Caputo derivatives in the Gateaux derivative of the functional relates Caputo with Riemann-Liouville derivatives.

Following [25], in the present work we obtain an Euler-Lagrange equation in integral form containing only Caputo derivatives and an Euler-Lagrange fractional differential equation only depending on Caputo derivatives.

The paper is organized as follows: some basic definitions of fractional derivatives are shown in section two. Section three presents the variational problems, whose Lagrangian depends on Caputo fractional derivative and the classical derivative. In section four, we obtain the Euler-Lagrange equations in integral form and the fractional Euler-Lagrange equation with only Caputo derivatives. In section five, a generalization is shown. We end with an illustrative example of the results of the paper, and with our conclusions.

\section{Preliminaries on Fractional Calculus}

In this section, we present some definitions and properties of the Caputo and Riemann-Liouville fractional calculus. For more details on the subject and applications, we refer the reader to [15, 24].

\section{Definition 1}

The left and right Riemann-Liouville fractional integral operators of order $\alpha \in \mathbb{R}_{+}$are defined, respectively, by

$$
{ }_{a} I_{x}^{\alpha}[f](x)=\frac{1}{\Gamma(\alpha)} \int_{a}^{x}(x-\xi)^{\alpha-1} f(\xi) d \xi
$$

and

$$
{ }_{x} I_{b}^{\alpha}[f](x)=\frac{1}{\Gamma(\alpha)} \int_{x}^{b}(\xi-x)^{\alpha-1} f(\xi) d \xi
$$

where $a, b \in \mathbb{R}$ with $a<b$. For $\alpha=0$, we set ${ }_{a} I_{x}^{0}={ }_{x} I_{b}^{0}=I$, the identity operator.

The Riemann-Liouville fractional integrals coincide with the classical definition of these in the case $n \in \mathbb{N}$. Moreover, in the case $\alpha \geq 1$ it is obvious that the integrals exist for any integrable function $f$ and for every $x \in[a, b]$. In the case $0<\alpha<1$ though, the situation is less clear at first sight. However, it is possible to prove the convergence (see [15]).

\section{Remark 1}

Let $n, m \in \mathbb{N}$, with $n>m$, we have

$$
\frac{d^{n}}{d x^{n}} \circ{ }_{a} I_{x}^{n-m}[f](x)=\frac{d^{m}}{d x^{m}}[f](x)
$$

This property motivates the following definition. 


\section{Definition 2}

The left and right Riemann-Liouville fractional derivative operators of order $\alpha \in \mathbb{R}_{+}$are defined, respectively, by

$$
{ }_{a} D_{x}^{\alpha}:=\frac{d^{n}}{d x^{n}} \circ{ }_{a} I_{x}^{n-\alpha}
$$

and

with $n=\lceil\alpha\rceil$, that is

$$
{ }_{x} D_{b}^{\alpha}:=(-1)^{n} \frac{d^{n}}{d x^{n}} \circ{ }_{x} I_{b}^{n-\alpha}
$$

$$
{ }_{a} D_{x}^{\alpha}[f](x)=\frac{1}{\Gamma(n-\alpha)} \frac{d^{n}}{d x^{n}} \int_{a}^{x}(x-\xi)^{n-1-\alpha} f(\xi) d \xi
$$

and

$$
{ }_{x} D_{b}^{\alpha}[f](x)=\frac{(-1)^{n}}{\Gamma(n-\alpha)} \frac{d^{n}}{d x^{n}} \int_{x}^{b}(\xi-x)^{n-1-\alpha} f(\xi) d \xi .
$$

Definition 3

The left and right Caputo fractional derivative operators of order $\alpha \in \mathbb{R}_{+}$are defined, respectively, by

$$
{ }_{a}^{C} D_{x}^{\alpha}:={ }_{a} I_{x}^{n-\alpha} \circ \frac{d^{n}}{d x^{n}}
$$

and

$$
{ }_{x}^{C} D_{b}^{\alpha}:={ }_{x} I_{b}^{n-\alpha} \circ(-1)^{n} \frac{d^{n}}{d x^{n}}
$$

with $n=\lceil\alpha\rceil$, that is

$$
{ }_{a}^{C} D_{x}^{\alpha}[f](x)=\frac{1}{\Gamma(n-\alpha)} \int_{a}^{x}(x-\xi)^{n-1-\alpha} \frac{d^{n}}{d \xi^{n}} f(\xi) d \xi
$$

and

$$
{ }_{x}^{C} D_{b}^{\alpha}[f](x)=\frac{(-1)^{n}}{\Gamma(n-\alpha)} \int_{x}^{b}(\xi-x)^{n-1-\alpha} \frac{d^{n}}{d \xi^{n}} f(\xi) d \xi .
$$

Now we will see some different properties of the Riemann-Liouville and Caputo derivatives.

\section{Remark 2}

Let $0<\alpha<1$. Assume that $f$ is such that ${ }_{a} D_{x}^{\alpha}[f],{ }_{x} D_{b}^{\alpha}[f],{ }_{a}^{C} D_{x}^{\alpha}[f]$ and ${ }_{x}^{C} D_{b}^{\alpha}[f]$ exist, then:

$$
{ }_{a}^{C} D_{x}^{\alpha}[f](x)={ }_{a} D_{x}^{\alpha}[f](x)-\frac{f(a)}{1-\alpha}(x-a)^{-\alpha}
$$

and

$$
{ }_{x}^{C} D_{b}^{\alpha}[f](x)={ }_{x} D_{b}^{\alpha}[f](x)-\frac{f(b)}{1-\alpha}(b-x)^{-\alpha}
$$

so we have that if $f(a)=0$ then

$$
{ }_{a}^{C} D_{x}^{\alpha}[f](x)={ }_{a} D_{x}^{\alpha}[f](x)
$$

and we have that if $f(b)=0$ then

$$
{ }_{x}^{C} D_{b}^{\alpha}[f](x)={ }_{x} D_{b}^{\alpha}[f](x) .
$$

Remark 3

An important difference between Riemann-Liouville derivatives and Caputo derivatives is that, being $\mathrm{K}$ an arbitrary constant, is

$$
{ }_{a}^{C} D_{x}^{\alpha} K=0, \quad{ }_{x}^{C} D_{b}^{\alpha} K=0,
$$

however

$$
{ }_{a} D_{x}^{\alpha} K=\frac{K}{\Gamma(1-\alpha)}(x-a)^{-\alpha}, \quad{ }_{x} D_{b}^{\alpha} K=\frac{K}{\Gamma(1-\alpha)}(b-x)^{-\alpha}
$$




$$
{ }_{a} D_{x}^{\alpha}(x-a)^{\alpha-1}=0, \quad{ }_{x} D_{b}^{\alpha}(b-x)^{\alpha-1}=0 .
$$

In this sense, the Caputo fractional derivatives are similar to the classical derivatives. In some cases it is easier to solve differential equations with Caputo derivatives instead of equations involving both Caputo and RiemannLiouville derivatives. Therefore in this work, we will state and prove equations with only derivatives of Caputo.

Theorem 1

Let $\alpha>0$ and $f \in L^{1}([a, b])$, then we have

$$
{ }_{a} D_{x a}^{\alpha} I_{x}^{\alpha}[f](x)=f(x), \quad{ }_{x} D_{b}^{\alpha}{ }_{x} I_{b}^{\alpha}[f](x)=f(x)
$$

and

$$
{ }_{a}^{C} D_{x a}^{\alpha} I_{x}^{\alpha}[f](x)=f(x), \quad{ }_{x}^{C} D_{b a}^{\alpha} I_{x}^{\alpha}[f](x)=f(x)
$$

almost everywhere.

Theorem 2

Let $0<\alpha<1$. Let $f \in C^{1}([a, b])$ and $g \in L^{1}([a, b])$. Then,

$$
\int_{a}^{b} g(x)_{a}^{C} D_{x}^{\alpha} f(x) d x=\int_{a}^{b} f(x){ }_{x} D_{b}^{\alpha} g(x) d x+\left.\left[{ }_{x} I_{b}^{1-\alpha} g(x) f(x)\right]\right|_{a} ^{b}
$$

and

$$
\int_{a}^{b} g(x)_{x}^{C} D_{b}^{\alpha} f(x) d x=\int_{a}^{b} f(x){ }_{a} D_{x}^{\alpha} g(x) d x-\left.\left[{ }_{a} I_{x}^{1-\alpha} g(x) f(x)\right]\right|_{a} ^{b} .
$$

Moreover, if $f(a)=f(b)=0$, we have that

$$
\int_{a}^{b} g(x){ }_{a}^{C} D_{x}^{\alpha} f(x) d x=\int_{a}^{b} f(x){ }_{x} D_{b}^{\alpha} g(x) d x
$$

and

$$
\int_{a}^{b} g(x)_{x}^{C} D_{b}^{\alpha} f(x) d x=\int_{a}^{b} f(x){ }_{a} D_{x}^{\alpha} g(x) d x .
$$

This theorem is known as Integration by parts and its proof is in [24].

\section{Fractional Variational Calculus with Classical Derivatives}

We begin with the following problem of the fractional calculus of variations: find a function $y \in{ }_{a}^{\alpha} E^{\prime}$ that optimizes (minimizes or maximizes) the functional

$$
J(y)=\int_{a}^{b} L\left(x, y, y^{\prime},{ }_{a}^{C} D_{x}^{\alpha} y\right) d x
$$

with a Lagrangian $L \in C^{1}\left([a, b] \times \mathbb{R}^{3}\right)$ and

$$
{ }_{a}^{\alpha} E^{\prime}=\left\{y:[a, b] \rightarrow \mathbb{R}: y \in C^{1}([a, b]),{ }_{a}^{C} D_{x}^{\alpha} y \in C([a, b])\right\}
$$

subject to the boundary conditions: $y(a)=y_{a}, y(b)=y_{b}$.

We now state the Euler-Lagrange equation for this problem, its proof is in [27].

Theorem 3

If $y$ is a local optimizer to the above problem, then $y$ satisfies the next Euler-Lagrange equation:

$$
\frac{\partial L}{\partial y}-\frac{d}{d x}\left(\frac{\partial L}{\partial y^{\prime}}\right)+{ }_{x} D_{b}^{\alpha} \frac{\partial L}{\partial_{a}^{C} D_{x}^{\alpha} y}=0 .
$$

Remark 4

We call these equations involving both Caputo and Riemann-Liouville derivatives. 


\section{Fractional Euler-Lagrange Equation with Only Caputo Derivatives}

You can find the following lemma in [25], made by Lazo M. and Torres D. (2013).

\section{Lemma 1}

Let $g$ be a differentiable function in $[a, b]$ with $g(a)=g(b)=0$, and let $f \in L^{1}([a, b])$ be such that there is a number $\epsilon \in(a, b]$ with $|f(x)| \leq c(x-a)^{\beta}$ for all $x \in[a, \epsilon]$, where $c>0$ and $\beta>-\alpha$ are constants. We have that,

$$
\text { if }{ }_{a} I_{b}^{\alpha}\left(f(x){ }_{a}^{C} D_{x}^{\alpha} g(x)\right)=0 \text {, then } f \equiv K
$$

where $K$ is a constant.

This lemma is known as the Dubois-Reymond Fundamental Lemma of the Fractional Calculus of Variations.

Remark 5

Using the definition of the Riemann-Liouville fractional integral (2), we can write the functional $J$ as

$$
J(y)=\int_{a}^{b} L\left(x, y, y^{\prime},{ }_{a}^{C} D_{x}^{\alpha} y\right) d x=\Gamma(\alpha)_{a} I_{b}^{\alpha}\left[(b-x)^{1-\alpha} L\left(x, y, y^{\prime},{ }_{a}^{C} D_{x}^{\alpha} y\right)\right] .
$$

Inspired on the work of [25], we have made the following generalization considering a Langrangian that depends on the Caputo derivatives and the classical derivative $y^{\prime}$.

Theorem 4

Let $J$ be the next functional

$$
J(y)=\int_{a}^{b} L\left(x, y, y^{\prime},{ }_{a}^{C} D_{x}^{\alpha} y\right) d x=\Gamma(\alpha){ }_{a} I_{b}^{\alpha}\left[(b-x)^{1-\alpha} L\left(x, y, y^{\prime},{ }_{a}^{C} D_{x}^{\alpha} y\right)\right]
$$

defined in the class of functions $y \in{ }_{a}^{\alpha} E^{\prime}$, and where we assume $L \in C^{1}\left([a, b) \times \mathbb{R}^{3}\right)$, differentiable with respect all of its arguments. If $y$ is an optimizer of $J$, then $y$ satisfies the following fractional Euler-Lagrange integral equation:

$$
{ }_{x} I_{b}^{\alpha} \frac{\partial L}{\partial y}-{ }_{x} I_{b}^{\alpha} \frac{d}{d x}\left(\frac{\partial L}{\partial y^{\prime}}\right)+\frac{\partial L}{\partial_{a}^{C} D_{x}^{\alpha} y}=\frac{K}{(b-x)^{1-\alpha}}
$$

for all $x \in[a, b)$, where $K$ is a constant.

Proof.: Let $y^{*}$ be an optimizer of $J$. We define the family of functions

$$
y(x)=y^{*}(x)+\epsilon \eta(x)
$$

where $\epsilon$ is a constant, and $\eta \in C^{1}([a, b])$ is an arbitrary function satisfying the boundary conditions $\eta(a)=$ $\eta(b)=0$. From (30), $\eta(a)=\eta(b)=0, y^{*}(a)=y_{a}$ and $y^{*}(b)=y_{b}$, we have that the function $y$ is admissible, that is, $y \in C^{1}([a, b]), y(a)=y_{a}$ and $y(b)=y_{b}$.

From now on, we denote $L[y]=L\left(x, y, y^{\prime},{ }_{a}^{C} D_{x}^{\alpha} y\right)$.

Because $y^{*}$ is an optimizer of the functional $J$, the Gateaux derivative $\delta J\left[y^{*}\right]$ needs to be identically null.

$$
\begin{aligned}
\delta J\left[y^{*}\right] & =\lim _{\epsilon \rightarrow 0} \frac{1}{\epsilon}\left(\int_{a}^{b} L[y] d x-\int_{a}^{b} L\left[y^{*}\right] d x\right) \\
& =\int_{a}^{b}\left(\eta(x) \frac{\partial L\left[y^{*}\right]}{\partial y^{*}}+\eta^{\prime}(x) \frac{\partial L\left[y^{*}\right]}{\partial y^{\prime *}}+{ }_{a}^{C} D_{x}^{\alpha} \eta(x) \frac{\partial L\left[y^{*}\right]}{\partial_{a}^{C} D_{x}^{\alpha} y^{*}}\right) d x=0 .
\end{aligned}
$$

We define $A=\int_{a}^{b}\left(\eta(x) \frac{\partial L\left[y^{*}\right]}{\partial y^{*}}\right) d x, B=\int_{a}^{b}\left(\eta^{\prime}(x) \frac{\partial L\left[y^{*}\right]}{\partial y^{\prime *}}\right) d x$ and $C=\int_{a}^{b}\left({ }_{a}^{C} D_{x}^{\alpha} \eta(x) \frac{\partial L\left[y^{*}\right]}{\partial{ }_{a}^{C} D_{x}^{\alpha} y^{*}}\right) d x$. Using the fact that ${ }_{x} D_{b}^{\alpha}{ }_{x} I_{b}^{\alpha} \equiv I$, the homogeneity conditions on $\eta$ and integration by parts we get

$$
A=\int_{a}^{b}\left(\eta(x) \frac{\partial L\left[y^{*}\right]}{\partial y^{*}}\right) d x=\int_{a}^{b}\left(\eta(x)_{x} D_{b}^{\alpha}{ }_{x} I_{b}^{\alpha} \frac{\partial L\left[y^{*}\right]}{\partial y^{*}}\right) d x=\int_{a}^{b}\left({ }_{a}^{C} D_{x}^{\alpha} \eta(x)_{x} I_{b}^{\alpha} \frac{\partial L\left[y^{*}\right]}{\partial y^{*}}\right) d x .
$$


Similarly,

$$
\begin{aligned}
B & =\int_{a}^{b}\left(\eta^{\prime}(x) \frac{\partial L\left[y^{*}\right]}{\partial y^{\prime *}}\right) d x=\left.\eta(x) \frac{\partial L\left[y^{*}\right]}{\partial y^{\prime *}}\right|_{a} ^{b}-\int_{a}^{b}\left(\eta(x) \frac{d}{d x} \frac{\partial L\left[y^{*}\right]}{\partial y^{\prime *}}\right) d x \\
& =-\int_{a}^{b}\left(\eta(x)_{x} D_{b}^{\alpha}{ }_{x}^{\alpha} I_{b}^{\alpha} \frac{d}{d x} \frac{\partial L\left[y^{*}\right]}{\partial y^{\prime *}}\right) d x=-\int_{a}^{b}\left({ }_{a}^{C} D_{x}^{\alpha} \eta(x)_{x} I_{b}^{\alpha} \frac{d}{d x} \frac{\partial L\left[y^{*}\right]}{\partial y^{\prime *}}\right) d x
\end{aligned}
$$

And we get,

$$
A+B+C=\int_{a}^{b}{ }_{a}^{C} D_{x}^{\alpha} \eta(x)\left[{ }_{x} I_{b}^{\alpha} \frac{\partial L\left[y^{*}\right]}{\partial y^{*}}-{ }_{x} I_{b}^{\alpha} \frac{d}{d x} \frac{\partial L\left[y^{*}\right]}{\partial y^{\prime *}}+\frac{\partial L\left[y^{*}\right]}{\partial_{a}^{C} D_{x}^{\alpha} y^{*}}\right] d x
$$

Writing this otherwise, we obtain for the first variation the following expression:

$$
\Gamma(\alpha){ }_{a} I_{b}^{\alpha}\left[{ }_{a}^{C} D_{x}^{\alpha} \eta(x)\left({ }_{x} I_{b}^{\alpha} \frac{\partial L\left[y^{*}\right]}{\partial y^{*}}-{ }_{x} I_{b}^{\alpha} \frac{d}{d x} \frac{\partial L\left[y^{*}\right]}{\partial y^{\prime *}}+\frac{\partial L\left[y^{*}\right]}{\partial_{a}^{C} D_{x}^{\alpha} y^{*}}\right)(b-x)^{1-\alpha}\right]=0 .
$$

We define

$$
f(x)=\left({ }_{x} I_{b}^{\alpha} \frac{\partial L\left[y^{*}\right]}{\partial y^{*}}-{ }_{x} I_{b}^{\alpha} \frac{d}{d x} \frac{\partial L\left[y^{*}\right]}{\partial y^{\prime *}}+\frac{\partial L\left[y^{*}\right]}{\partial{ }_{a}^{C} D_{x}^{\alpha} y^{*}}\right)(b-x)^{1-\alpha}
$$

As $L \in C^{1}\left([a, b) \times \mathbb{R}^{3}\right)$, we have that $f$ is bounded in a sufficiently small neighborhood of $a$ and taking $\beta=0>-\alpha$, the hypotheses of the Lemma are verified so it exists $K$ constant such that

$$
\left({ }_{x} I_{b}^{\alpha} \frac{\partial L\left[y^{*}\right]}{\partial y^{*}}-{ }_{x} I_{b}^{\alpha} \frac{d}{d x} \frac{\partial L\left[y^{*}\right]}{\partial y^{\prime *}}+\frac{\partial L\left[y^{*}\right]}{\partial_{a}^{C} D_{x}^{\alpha} y^{*}}\right)(b-x)^{1-\alpha}=K
$$

and we obtain the desired equation,

$$
{ }_{x} I_{b}^{\alpha} \frac{\partial L}{\partial y}-{ }_{x} I_{b}^{\alpha} \frac{d}{d x}\left(\frac{\partial L}{\partial y^{\prime}}\right)+\frac{\partial L}{\partial_{a}^{C} D_{x}^{\alpha} y}=\frac{K}{(b-x)^{1-\alpha}}
$$

In the following theorem we will see a new version of the Euler-Lagrange equation. Its proof follows the same guidelines of the paper [25].

\section{Theorem 5}

Let $y$ be an optimizer of $J$ in ${ }_{a}^{\alpha} E^{\prime}$ with $L \in C^{2}\left([a, b] \times \mathbb{R}^{3}\right)$ subject to boundary conditions $y(a)=y_{a}, y(b)=y_{b}$, then $y$ satisfies the fractional Euler-Lagrange differential equation

$$
\frac{\partial L}{\partial y}-\frac{d}{d x}\left(\frac{\partial L}{\partial y^{\prime}}\right)+{ }_{x}^{C} D_{b}^{\alpha} \frac{\partial L}{\partial{ }_{a}^{C} D_{x}^{\alpha} y}=0 .
$$

\subsection{The Fractional Isoperimetric Problem}

We now consider the following isoperimetric problem of the fractional calculus of variations: find a function $y \in{ }_{a}^{\alpha} E^{\prime}$ that optimizes the functional

$$
J(y)=\int_{a}^{b} L\left(x, y, y^{\prime},{ }_{a}^{C} D_{x}^{\alpha} y\right) d x
$$

with a Lagrangian $L \in C^{1}\left([a, b] \times \mathbb{R}^{3}\right)$ and

$$
{ }_{a}^{\alpha} E^{\prime}=\left\{y:[a, b] \rightarrow \mathbb{R}: y \in C^{1}([a, b]),{ }_{a}^{C} D_{x}^{\alpha} y \in C([a, b])\right\}
$$


subject to the boundary conditions $y(a)=y_{a}, y(b)=y_{b}$, and an integral constraint

$$
I(y)=\int_{a}^{b} G\left(x, y, y^{\prime},{ }_{a}^{C} D_{x}^{\alpha} y\right) d x=c,
$$

where $G \in C^{1}\left([a, b] \times \mathbb{R}^{3}\right)$ and $c \in \mathbb{R}$.

Such variational problems have found a broad class of important applications throughout the centuries, with numerous useful implications in astronomy, geometry, algebra, and engineering. For references and recent advancements on the subject, we refer the reader to $[16,30]$.

We now state the Euler-Lagrange equation for this problem, its proof is in [27].

\section{Theorem 6}

If $y$ is a local optimizer to the above problem, and suppose further that $y$ does not anulate the Gateaux's derivative of the functional $I$. Then there exists a constant $\lambda$ such that $y$ satisfies the next Euler-Lagrange equation:

$$
\frac{\partial F}{\partial y}-\frac{d}{d x}\left(\frac{\partial F}{\partial y^{\prime}}\right)+{ }_{x} D_{b}^{\alpha} \frac{\partial F}{\partial{ }_{a}^{C} D_{x}^{\alpha} y}=0
$$

where $F=L+\lambda G$.

\section{Theorem 7}

Let $J$ and $I$ be the above functionals, defined in the class of functions $y \in{ }_{a}^{\alpha} E^{\prime}$, and where $L, G \in C^{1}\left([a, b) \times \mathbb{R}^{3}\right)$ are differentiable with respect all of its arguments. If $y$ is an optimizer of $J$ and it does not anulate the Gateaux's derivative of the functional $I$ then $y$ satisfies the following fractional Euler-Lagrange integral equation:

$$
{ }_{x} I_{b}^{\alpha} \frac{\partial F}{\partial y}-{ }_{x} I_{b}^{\alpha} \frac{d}{d x}\left(\frac{\partial F}{\partial y^{\prime}}\right)+\frac{\partial F}{\partial_{a}^{C} D_{x}^{\alpha} y}=\frac{K}{(b-x)^{1-\alpha}}
$$

for all $x \in[a, b)$, where $K$ is a constant and $F=L+\lambda G$.

Proof:: Consider $\eta_{1}, \eta_{2} \in{ }_{a}^{\alpha} E^{\prime}$ two functions such that $\eta_{1}(a)=\eta_{1}(b)=\eta_{2}(a)=\eta_{2}(b)=0$, and $\left|\epsilon_{1}\right| \ll 1$, $\left|\epsilon_{2}\right| \ll 2$.

We define

$$
j\left(\epsilon_{1}, \epsilon_{2}\right)=\int_{a}^{b} L\left(x, y+\epsilon_{1} \eta_{1}+\epsilon_{2} \eta_{2}, y^{\prime}+\epsilon_{1} \eta_{1}^{\prime}+\epsilon_{2} \eta_{2}^{\prime},{ }_{a}^{C} D_{x}^{\alpha} y+\epsilon_{1}{ }_{a}^{C} D_{x}^{\alpha} \eta_{1}+\epsilon_{2}{ }_{a}^{C} D_{x}^{\alpha} \eta_{2}\right) d x
$$

and

$$
i\left(\epsilon_{1}, \epsilon_{2}\right)=\int_{a}^{b} g\left(x, y+\epsilon_{1} \eta_{1}+\epsilon_{2} \eta_{2}, y^{\prime}+\epsilon_{1} \eta_{1}^{\prime}+\epsilon_{2} \eta_{2}^{\prime},{ }_{a}^{C} D_{x}^{\alpha} y+\epsilon_{1}{ }_{a}^{C} D_{x}^{\alpha} \eta_{1}+\epsilon_{2}{ }_{a}^{C} D_{x}^{\alpha} \eta_{2}\right) d x-c
$$

As $y$ does not anulate the Gateaux's derivative of the functional $I$, then there is a function $\eta_{2}$ such that,

$$
\left.\frac{\partial i}{\partial \epsilon_{2}}\right|_{(0,0)} \neq 0
$$

and using the implicit function theorem, there exists a function $\epsilon_{2}(.) \in C^{1}$, defined in a neighborhood of zero such that

$$
i\left(\epsilon_{1}, \epsilon_{2}\left(\epsilon_{1}\right)\right)=0 .
$$

Applying the rule of Lagrange multipliers, there exists $\lambda$ constant so that $\nabla(j(0,0)+\lambda i(0,0))=(0,0)$. Therefore,

$$
\underbrace{\frac{\partial j}{\partial \epsilon_{1}}(0,0)+\lambda \frac{\partial i}{\partial \epsilon_{1}}(0,0)}_{(A)}=0 \text { and } \underbrace{\frac{\partial j}{\partial \epsilon_{2}}(0,0)+\lambda \frac{\partial i}{\partial \epsilon_{2}}(0,0)}_{(B)}=0 .
$$


Then, we have

$$
\begin{aligned}
& \frac{\partial j}{\partial \epsilon_{1}}(0,0)=\int_{a}^{b} \frac{\partial L}{\partial y} \eta_{1}+\frac{\partial L}{\partial y^{\prime}} \eta_{1}^{\prime}+\frac{\partial L}{\partial{ }_{a}^{C} D_{x}^{\alpha} y}{ }_{a}^{C} D_{x}^{\alpha} \eta_{1} d x \\
& \frac{\partial i}{\partial \epsilon_{1}}(0,0)=\int_{a}^{b} \frac{\partial g}{\partial y} \eta_{1}+\frac{\partial g}{\partial y^{\prime}} \eta_{1}^{\prime}+\frac{\partial g}{\partial{ }_{a}^{C} D_{x}^{\alpha} y}{ }_{a}^{C} D_{x}^{\alpha} \eta_{1} d x
\end{aligned}
$$

Thus,

$$
(A)=\int_{a}^{b} \frac{\partial L}{\partial y} \eta_{1}+\lambda \frac{\partial g}{\partial y} \eta_{1}+\frac{\partial L}{\partial y^{\prime}} \eta_{1}^{\prime}+\lambda \frac{\partial g}{\partial y^{\prime}} \eta_{1}^{\prime}+\frac{\partial L}{\partial{ }_{a}^{C} D_{x}^{\alpha} y} \underset{a}{C} D_{x}^{\alpha} \eta_{1}+\lambda \frac{\partial g}{\partial{ }_{a}^{C} D_{x}^{\alpha} y}{ }_{a}^{C} D_{x}^{\alpha} \eta_{1} d x
$$

defining $F=L+\lambda g$, we can write (A) as:

$$
(A)=\int_{a}^{b} \frac{\partial F}{\partial y} \eta_{1}+\frac{\partial F}{\partial y^{\prime}} \eta_{1}^{\prime}+\frac{\partial F}{\partial{ }_{a}^{C} D_{x}^{\alpha} y}{ }_{a}^{C} D_{x}^{\alpha} \eta_{1} d x .
$$

Similarly,

$$
(B)=\int_{a}^{b} \frac{\partial F}{\partial y} \eta_{2}+\frac{\partial F}{\partial y^{\prime}} \eta_{2}^{\prime}+\frac{\partial F}{\partial_{a}^{C} D_{x}^{\alpha} y}{ }_{a}^{C} D_{x}^{\alpha} \eta_{2} d x
$$

Integrating by parts as in Theorem (4), we obtain:

$$
\begin{aligned}
& (A)=\int_{a}^{b}{ }_{a}^{C} D_{x}^{\alpha} \eta_{1}(x)\left[{ }_{x} I_{b}^{\alpha} \frac{\partial F}{\partial y}-{ }_{x} I_{b}^{\alpha} \frac{d}{d x} \frac{\partial F}{\partial y^{\prime}}+\frac{\partial F}{\partial\left({ }_{a}^{C} D_{x}^{\alpha} y\right)}\right] d x=0, \\
& (B)=\int_{a}^{b}{ }_{a}^{C} D_{x}^{\alpha} \eta_{2}(x)\left[{ }_{x} I_{b}^{\alpha} \frac{\partial F}{\partial y}-{ }_{x} I_{b}^{\alpha} \frac{d}{d x} \frac{\partial F}{\partial y^{\prime}}+\frac{\partial F}{\partial\left({ }_{a}^{C} D_{x}^{\alpha} y\right)}\right] d x=0 .
\end{aligned}
$$

Writing this differently,

$$
\begin{aligned}
& (A)=\Gamma(\alpha){ }_{a} I_{b}^{\alpha}\left[{ }_{a}^{C} D_{x}^{\alpha} \eta_{1}(x)\left({ }_{x} I_{b}^{\alpha} \frac{\partial F}{\partial y}-{ }_{x} I_{b}^{\alpha} \frac{d}{d x} \frac{\partial F}{\partial y^{\prime}}+\frac{\partial F}{\partial\left({ }_{a}^{C} D_{x}^{\alpha} y\right)}\right)(b-x)^{1-\alpha}\right]=0 \\
& (B)=\Gamma(\alpha){ }_{a} I_{b}^{\alpha}\left[{ }_{a}^{C} D_{x}^{\alpha} \eta_{2}(x)\left({ }_{x} I_{b}^{\alpha} \frac{\partial F}{\partial y}-{ }_{x} I_{b}^{\alpha} \frac{d}{d x} \frac{\partial F}{\partial y^{\prime}}+\frac{\partial F}{\partial\left({ }_{a}^{C} D_{x}^{\alpha} y\right)}\right)(b-x)^{1-\alpha}\right]=0 .
\end{aligned}
$$

Note that if we proceed analogously to Theorem (4) and the condition (35) we get the same result

$$
{ }_{x} I_{b}^{\alpha} \frac{\partial F}{\partial y}-{ }_{x} I_{b}^{\alpha} \frac{d}{d x}\left(\frac{\partial F}{\partial y^{\prime}}\right)+\frac{\partial F}{\partial_{a}^{C} D_{x}^{\alpha} y}=\frac{K}{(b-x)^{1-\alpha}}
$$

for all $x \in[a, b)$, where $K$ is a constant.

In the following theorem we will see a new version of the Euler-Lagrange equation on isoperimetric problems. Its proof follows the same guidelines of paper [25].

\section{Theorem 8}

Let $y$ be an optimizer of $J$ in ${ }_{a}^{\alpha} E^{\prime}$ with $L, G \in C^{2}\left([a, b] \times \mathbb{R}^{3}\right)$ subject to boundary conditions $y(a)=y_{a} y(b)=y_{b}$, then if $y$ does not anulate the Gateaux's derivative of the functional $I$, it satisfies the fractional Euler-Lagrange differential equation:

$$
\frac{\partial F}{\partial y}-\frac{d}{d x}\left(\frac{\partial F}{\partial y^{\prime}}\right)+{ }_{x}^{C} D_{b}^{\alpha} \frac{\partial F}{\partial_{a}^{C} D_{x}^{\alpha} y}=0
$$

where $F=L+\lambda G$. 


\section{A Lagrangian with classical higher order derivatives}

Let us consider the following problem: find a function $y \in{ }_{a}^{\alpha} E^{n}$ that optimizes the functional

$$
J[y]=\int_{a}^{b} L\left(x, y, y^{\prime}, y^{\prime \prime}, \ldots, y^{n},{ }_{a}^{C} D_{x}^{\alpha} y\right) d x
$$

with a Lagrangian $L \in C^{1}\left([a, b] \times \mathbb{R}^{n+2}\right)$ and

$$
{ }_{a}^{\alpha} E^{n}=\left\{y:[a, b] \rightarrow \mathbb{R}: y \in C^{n}([a, b]),{ }_{a}^{C} D_{x}^{\alpha} y \in C([a, b])\right\}
$$

subject to the boundary conditions:

$$
y(a)=y_{a}, y(b)=y_{b}, y^{\prime}(a)=y_{a}^{\prime}, y^{\prime}(b)=y_{b}^{\prime}, \ldots y^{(n-1)}(a)=y_{a}^{(n-1)}, y^{(n-1)}(b)=y_{b}^{(n-1)} .
$$

Theorem 9

Let $J$ be the following functional

$$
J(y)=\int_{a}^{b} L\left(x, y, y^{\prime}, y^{\prime \prime}, \ldots, y^{(n)},{ }_{a}^{C} D_{x}^{\alpha} y\right) d x=\Gamma(\alpha){ }_{a} I_{b}^{\alpha}\left[(b-x)^{1-\alpha} L\left(x, y, y^{\prime}, y^{\prime \prime}, \ldots, y^{(n)},{ }_{a}^{C} D_{x}^{\alpha} y\right)\right]
$$

defined in the class of functions $y \in{ }_{a}^{\alpha} E^{n}$, and where we assume $L \in C^{1}\left([a, b) \times \mathbb{R}^{n+2}\right)$, differentiable with respect all of its arguments. If $y$ is an optimizer of $J$, then $y$ satisfies the following fractional Euler-Lagrange integral equation:

$$
{ }_{x} I_{b}^{\alpha} \frac{\partial L}{\partial y}+\sum_{j=1}^{n}(-1)^{j}{ }_{x} I_{b}^{\alpha} \frac{d^{j}}{d x^{j}}\left(\frac{\partial L}{\partial y^{(j)}}\right)+\frac{\partial L}{\partial{ }_{a}^{C} D_{x}^{\alpha} y}=\frac{K}{(b-x)^{1-\alpha}}
$$

for all $x \in[a, b)$, where $K$ is a constant.

Theorem 10

Let $y$ be an optimizer of $J$ in ${ }_{a}^{\alpha} E^{n}$ with $L \in C^{2}\left([a, b] \times \mathbb{R}^{n+2}\right)$ subject to boundary conditions (38), then $y$ satisfies the fractional Euler-Lagrange differential equation

$$
\frac{\partial L}{\partial y}+\sum_{j=1}^{n}(-1)^{j}{ }_{x} I_{b}^{\alpha} \frac{d^{j}}{d x^{j}}\left(\frac{\partial L}{\partial y^{(j)}}\right)+{ }_{x}^{C} D_{b}^{\alpha} \frac{\partial L}{\partial{ }_{a}^{C} D_{x}^{\alpha} y}=0 .
$$

Remark 6

The above theorems can be performed analogous to isoperimetric problems. Its proofs are similar to the case with $n=1$.

\section{An Example}

Consider the following fractional isoperimetric problem $J(y)=\int_{0}^{1}\left[y(x) y^{\prime}(x)+\left({ }_{0}^{C} D_{x}^{\alpha}[y]\right)^{2}(x)\right] d x, I(y)=$ $\int_{0}^{1}[y(x)] d x=1$, and the boundary conditions $y(0)=0, y(1)=0$.

Then, $F=y(x) y^{\prime}(x)+\left({ }_{0}^{C} D_{x}^{\alpha}[y]\right)^{2}(x)+\lambda y(x)$, and the Euler-Lagrange equation (using only Caputo derivatives) states

$$
{ }_{x}^{C} D_{1}^{\alpha}\left[{ }_{0}^{C} D_{x}^{\alpha}[y]\right](x)=-\frac{\lambda}{2} .
$$

Using the property

$$
{ }_{x}^{C} D_{1}^{\alpha}\left[(1-x)^{\beta}\right]=\frac{\Gamma(1+\beta)}{\Gamma(1+\beta-\alpha)}(1-x)^{\beta-\alpha}
$$


we obtain

$$
{ }_{0}^{C} D_{x}^{\alpha}[y](x)=-\frac{\lambda}{2} \frac{1}{\Gamma(1+\alpha)}(1-x)^{\alpha}+c_{1}
$$

We can write

$$
-\frac{\lambda}{2} \frac{1}{\Gamma(1+\alpha)}(1-x)^{\alpha}=-\frac{\lambda}{2} \frac{1}{\Gamma(1+\alpha)} \sum_{n=0}^{\infty} \frac{(-1)^{n} \prod_{j=0}^{n-1}(\alpha-j)}{n !} x^{n} .
$$

And consider

$$
{ }_{0}^{C} D_{x}^{\alpha}\left[x^{\beta}\right]=\frac{\Gamma(1+\beta)}{\Gamma(1+\beta-\alpha)} x^{\beta-\alpha}
$$

to obtain the solution

$$
y(x)=-\frac{\lambda}{2} \frac{1}{\Gamma(1+\alpha)} \sum_{n=0}^{\infty} \frac{(-1)^{n} \Gamma(\alpha)}{n ! \Gamma(\alpha-n+1)} \frac{\Gamma(1+n)}{\Gamma(1+n+\alpha)} x^{n+\alpha}+\widetilde{c}_{1} x^{\alpha}+c_{2} .
$$

Using the definition of the Hypergeometric function of parameters $a, b$ and $c$ :

$$
{ }_{2} F_{1}(a, b, c, x)=\sum_{n=0}^{\infty} \frac{\Gamma(a+n) \Gamma(b+n) \Gamma(c)}{\Gamma(a) \Gamma(b) \Gamma(c+n)} \frac{x^{n}}{n !}
$$

and the property

$$
\frac{(-1)^{n} \Gamma(\alpha)}{\Gamma(\alpha-n+1)}=\frac{\Gamma(n-\alpha)}{\alpha \Gamma(-\alpha)}
$$

we can write the solution as

$$
y(x)=-\frac{\lambda}{2 \alpha} \frac{x^{\alpha}}{\Gamma(1+\alpha)^{2}}{ }_{2} F_{1}(1,-\alpha, 1+\alpha, x)+\widetilde{c}_{1} x^{\alpha}+c_{2} .
$$

Taking into account that $y(0)=0, y(1)=0$ and $I(y)=\int_{0}^{1}[y(x)] d x=1$, we obtain

$$
y(x)=(\alpha+1)(2 \alpha+1)\left[2{ }_{2} F_{1}(1,-\alpha, 1+\alpha, x)-1\right] x^{\alpha} .
$$

When taking the limit $\alpha \rightarrow 1$, we have $y(x)=-6 x^{2}+6 x$, which is the solution to the classical problem

$$
J(y)=\int_{0}^{1}\left[y(x) y^{\prime}(x)+y^{\prime 2}(x)\right] d x
$$

subject to

$$
I(y)=\int_{0}^{1}[y(x)] d x=1, y(0)=0 \text { and } y(1)=0 .
$$

\section{Conclusion}

The generalized version of the fundamental lemma of the variational calculus of DuBois-Reymond enabled us to prove a fractional Euler-Lagrange equation in integral form only containing fractional derivatives of Caputo's type. We have done this for variational and isoperimetric problems in which the Lagrangian depends not only on the fractional derivatives of Caputo but also on derivatives in the classical sense. Furthermore, we also showed that when the Lagrangian is a $C^{2}$ function, we can obtain a fractional Euler-Lagrange differential equation only depending on Caputo derivatives. The advantage of this is that Caputo derivatives are superior to Riemann-Liouville derivatives in some cases. Finally, we obtained an exact solution of a particular isoperimetric problem, where the classical solution is recovered when $\alpha \rightarrow 1$. 


\section{Acknowledgement}

This work has been partially supported by the projects ING428 'Optimización y problemas de equilibrio' and ING506 'Cálculo variacional y control óptimo fraccionario' of Facultad de Ciencias Exactas, Ingenierła y Agrimensura, Universidad Nacional de Rosario. The first author was also supported by CONICET through a PhD fellowship.

\section{REFERENCES}

1. O.P. Agrawal, Formulation of Euler-Lagrange equations for fractional variational problems, Journal of Mathematical Analysis and Applications 272(1), pp368-379, 2002.

2. O.P. Agrawal, Generalized Euler-Lagrange equations and transversality conditions for FVPs in terms of Caputo derivative, Journal of Vibration and Control 13(9-10), pp1217-1237, 2007.

3. R. Almeida and D. Torres, Neccesary and sufficient conditions for the fractional calculus of variations with Caputo derivatives, Communications in Nonlinear Science and Numerical Simulation 16 (3), pp1490-1500, 2011.

4. R. Almeida and D. Torres, Leitmann's direct method for fractional optimization problems, Applied Mathematics and Computation 217, no. 3, pp956-962, 2010.

5. R. Almeida, R. Ferreira and D. Torres, Isoperimetric problems of the calculus of variations with fractional derivatives, Acta Mathematica Scientia, Volume 32, Issue 2, pp619-630, 2012.

6. R. Almeida, A. Malinowska and D. Torres, Fractional Euler-Lagrange differential equation via Caputo derivatives, Fractional Dynamics and Control,Part 2, pp109-118, 2012.

7. R. Almeida, A. Malinowska and D. Torres, A fractional calculus of variations for multiple integrals with application to vibrating string, Journal of Mathematical Physics 51, no. 3, 033503, pp12, 2010.

8. R. Almeida, S. Pooseh and D. Torres, Computational Methods in the Fractional Calculus of Variations, WORLD SCIENTIFIC Imperial College Press, London, 2015.

9. T. Apostol, Calculus, EDITORIAL REVERTÉ, Barcelona, Vol.2, 2 Ed.1967.

10. M. Barrios, A. Lombardi and G. Reyero, Método numérico de tipo L1 para problemas variacionales fraccionarios, AMCA, Mecánica Computacional Vol XXXVI, pp1089-1098, 2018.

11. N. Bastos, Calculus of variations involving Caputo-Fabrizio fractional differentiation, Statistics, Optimization and Information Computing, Vol. 6, pp12C-21, March 2018.

12. N. Bastos, R. Ferreira and D. Torres, Neccesary optimality conditions for fractional difference problems of the calculus of variations, Discrete and Continuous Dynamical Systems 29, no. 2, pp417-437, 2011.

13. N. Bastos, R. Ferreira and D. Torres, Discrete-time fractional variational problems, Signal Process 91, no. 3, pp513-524, 2011.

14. M. Caputo and F. Mainardi Linear models of dissipation in anelastic solids, La Rivista del Nuovo Cimento 1, pp161-198, 1971.

15. K. Diethelm, The analysis of fractional differential equations, SPRINGER, 2004.

16. R. Ferreira and D. Torres, Isoperimetric problems of the calculus of variations on time scales, in Nonlinear Analysis and Optimition II (eds: A.Leizarowitz B. S. Mordukhovich, I. Shafrir, And A.J.Zaslavski), Contemporary Mathematics, vol.514, pp123-131, 2010.

17. A. Ferrari and E. Santillan Marcus, Study of a fractional-order model for HIV infection of CD4+ T-cells with treatment, Journal of Fractional Calculus and Applications, Vol. 11(2), pp12-22, 2020.

18. G. Frederico and D. Torres, Fractional Noether's theorem in the Riesz-Caputo sense, Applied Mathematics and Computation 217, no. 3, pp1023-1033, 2010.

19. D. Goos and G. Reyero, Mathematical analysis of a Cauchy problem for the time-fractional diffusion-wave equation with $\alpha \in(0,2)$, Journal Fourier Analysis Applications, DOI 10.1007/s00041-017-9527-9, 2017.

20. D. Goos, G. Reyero, S. Rosacani and E. Santillan Marcus, On the initial-boundary-value problem for the time-fractional diffusion equation on the real positive semiaxis, International Journal of Differential Equations, Vol. 2015, ID 439419, 14 pages, DOI 1155/2015/439419, 2015.

21. R. Herrmann, Fractional Calculus: an Introduction for Physicist, WORLD SCIENTIFIC, Singapore, 2011.

22. R. Hilfer, Applications of Fractional Calculus in Physics, WORLD SCIENTIFIC, River Edge, NJ, 2000.

23. S. Jahanshahi and D. Torres, A simple accurate method for solving fractional variational and optimal control problems, Journal of Optimization Theory and Applications 174, no. 1, pp156-175, 2017.

24. A. Kilbas, H. Srivastava and J. Trujillo, Theory and Applications of Fractional Differential Equations, ELSEVIER, Amsterdan, 2006.

25. M. Lazo and D. Torres, The DuBois-Reymond fundamental lemma of the fractional calculus of variations and an Euler-Lagrange equation involving only derivatives of Caputo, Journal of Optimization Theory and Applications 156, pp56-67, 2013.

26. A. Malinowska, T. Odzijewicz and D. Torres, Advances methods in the fractional calculus of variations, SPRINGER, 2015.

27. A. Malinowska, T. Odzijewicz and D. Torres, Fractional Variational Calculus with Classical and Combined Caputo Derivatives, Nonlinear Analysis 75, Nro. 3, pp1507-1515, 2012.

28. A. Malinowska and D. Torres, Generalized natural boundary conditions for fractional variational problems in terms of the Caputo derivative, Computers \& Mathematics with Applications 59(9), pp3110-3116, 2010.

29. A. Malinowska and D. Torres, Introduction to the Fractional Calculus of Variations, IMPERIAL COLLEGE PRESS/WORLD SCIENTIFIC, London, Singapore, 2012.

30. A. Malinowska and D. Torres, Necessary and sufficient conditions for local Pareto optimality on time scales, Journal of Mathematical Sciences, pp803-810, 2009. 
31. T. Odzijewicz and D. Torres, Calculus of variations with fractional and classical derivatives, Proceedings of FDA'10, The 4th IFAC Workshop on Fractional Differentiation and its Applications, Badajoz, Spain, article no. FDA10-076, pp5, 2010.

32. K. Oldham and J. Spanier, The Fractional Calculus, ACADEMIC PRESS, New York, 1974.

33. I. Podlubny, Fractional differential equations. An introduction to fractional derivatives, fractional differential equations, to methods of their solution and some their applications, ACADEMIC PRESS, San Diego-New York- London, 1999.

34. B. Ross, S. Samko and E. Love, Functions that have no first order derivative might have fractional derivatives of all orders less than one, Real Analysis Exchange 20, no. 1, pp140-157, 1994.

35. B. Van Brunt, The calculus of variations, SPRINGER, 2004. 